\section{A rare case of high grade myxoinflammatory fibroblastic sarcoma of the neck with PRAME immuno-expression: a potential pitfall}

To the Editor,

Myxoinflammatory fibroblastic sarcomas (MIFS) are classically low grade soft tissue tumours of acral sites. ${ }^{1-4}$ The histology is striking, with fibrohistiocytic tumour cells set in a myxoinflammatory background which display virocyte- or Reed-Sternberg-like features, and which focally mimic the appearance of lipoblasts and ganglion cells. The molecular features have been characterised, including a $\mathrm{t}(1 ; 10)(\mathrm{p} 22 ; \mathrm{q} 24)$ translocation with TGFBR3/MGEA5 rearrangement. ${ }^{5-7}$ Low grade MIFS can be locally aggressive and are prone to local recurrence if incompletely excised.
High grade MIFS are extremely rare variants, which are often central (non-acral) in location, can grow to a significant size before becoming clinically apparent, and possess metastatic potential. ${ }^{8}$ Rare cases have been described in the head and neck. ${ }^{9,10}$ Interestingly, high grade MIFS have been described to show immunoreactivity to the PRAME (preferentially expressed antigen in melanoma) antibody, ${ }^{8}$ a new marker for melanocytic malignancy. ${ }^{11}$ Appreciation of this fact is important as PRAME-positive high grade MIFS can be mistaken for malignant melanoma on limited biopsies. We report a rare case of a high grade MIFS of the neck, which on initial biopsy presented a diagnostic challenge.

Our patient was a 70-year-old Caucasian male who presented with a painless palpable firm right neck mass which had become noticeable. He was otherwise well. His past medical history included ischaemic heart disease and childhood adenoid-tonsillectomy. There was no significant family history of neoplasia.
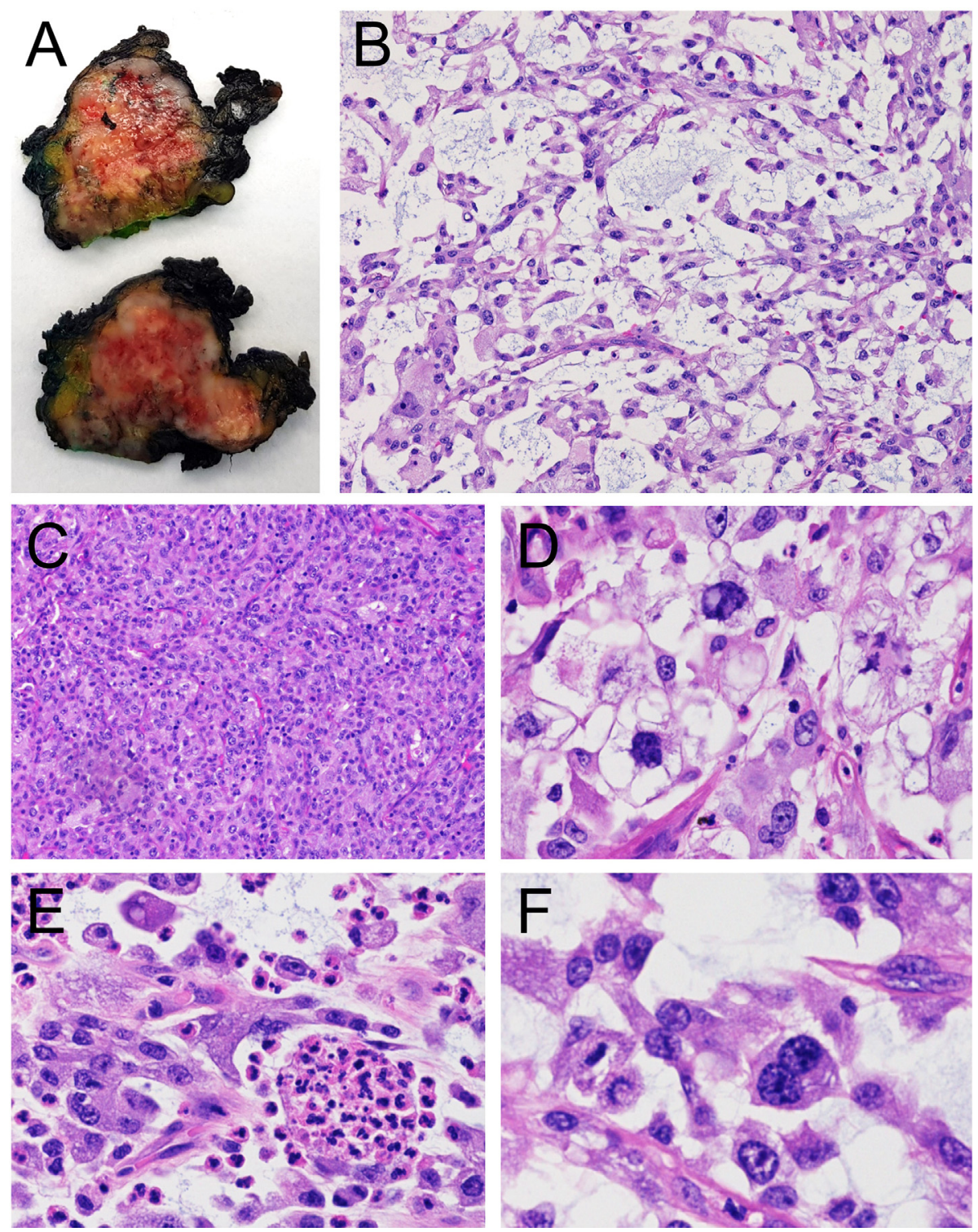

Fig. 1 (A) Macroscopic appearance. (B) Low power view of myxoinflammatory fibroblastic sarcoma showing areas with myxoid stroma and (C) dedifferentiation. (D) High power views of pseudolipoblasts, (E) emperipolesis, and (F) Reed-Sternberg-like cell. 
FDG PET/CT imaging detected a $39 \mathrm{~mm}$ necrotic right level III neck node. Imaging guided core biopsies of the main tumour showed an undifferentiated/poorly differentiated high grade malignancy with epithelioid, plasmacytoid, and rhabdoid morphology. A comprehensive immunohistochemistry panel was inconclusive, significant only for PRAME positivity which was strong and diffuse, with concomitant negativity for Melan A, HMB45, and SOX10. A provisional diagnosis of a high grade poorly differentiated malignancy was rendered, with the possibility of metastatic melanoma raised as a diagnostic differential.

The patient was referred for surgical excision with a modified radical right neck dissection. Intraoperatively, a mass was noted in the low right neck (centred on levels IV and $\mathrm{Vb}$ ) abutting the right phrenic and subclavian nerves. The tumour and right neck dissection were submitted for histopathology, and viable tissue was sent for cytogenetic analysis.

Specimen grossing post-formalin fixation revealed a $40 \mathrm{~mm}$ ovoid tumour mass with firm tan cut surfaces (Fig. 1A) surrounded by fat and some skeletal muscle. No cystic change or extensively necrotic areas were seen. Microscopically, the tumour was an un-encapsulated peripherally infiltrative tumour with myxoid stroma and a lymphoplasmacytic inflammatory background (Fig. 1B). The tumour cells were spindled and epithelioid, arranged in sheets and lace-like reticular formations with a lobulated configuration on scanning magnification. Characteristic virocyte-like and Reed-Sternberg-like cells were identified (Fig. 1F, 2B-D), as well as focal areas with pseudolipoblasts (Fig. 1D) and ganglion-like cells (Fig. 2A). There was striking neutrophilic emperipolesis by large multinucleated cells with voluminous cytoplasm (Fig. 1E). Poorly differentiated areas with increased mitotic activity were noted at the tumour periphery where hypercellular sheets of epitheloid and sarcomatoid cells with plasmacytoid and rhabdoid cytology were seen infiltrating into surrounding adipose tissue (Fig. 1C; digital slide https://myapi.pathomation.com/slide/ share?s=UhR7ENjd2ms).

The tumour cells were immuno-positive for CD68, CD31, CyclinD1, and PRAME (Fig. 2G; digital slide https://myapi. pathomation.com/slide/share?s=iIAUtrfYAxV). Melanocytic (Melan A, MiTF, HMB45 and SOX10) and cytokeratin (CK8/18, AE1/AE3 and CK5/6) markers were negative. PRAME positivity was limited to the high grade sarcomatoid areas with crisp nuclear staining. Further negative stains include: desmin, EMA, CD34, ERG, p16, myogenin,
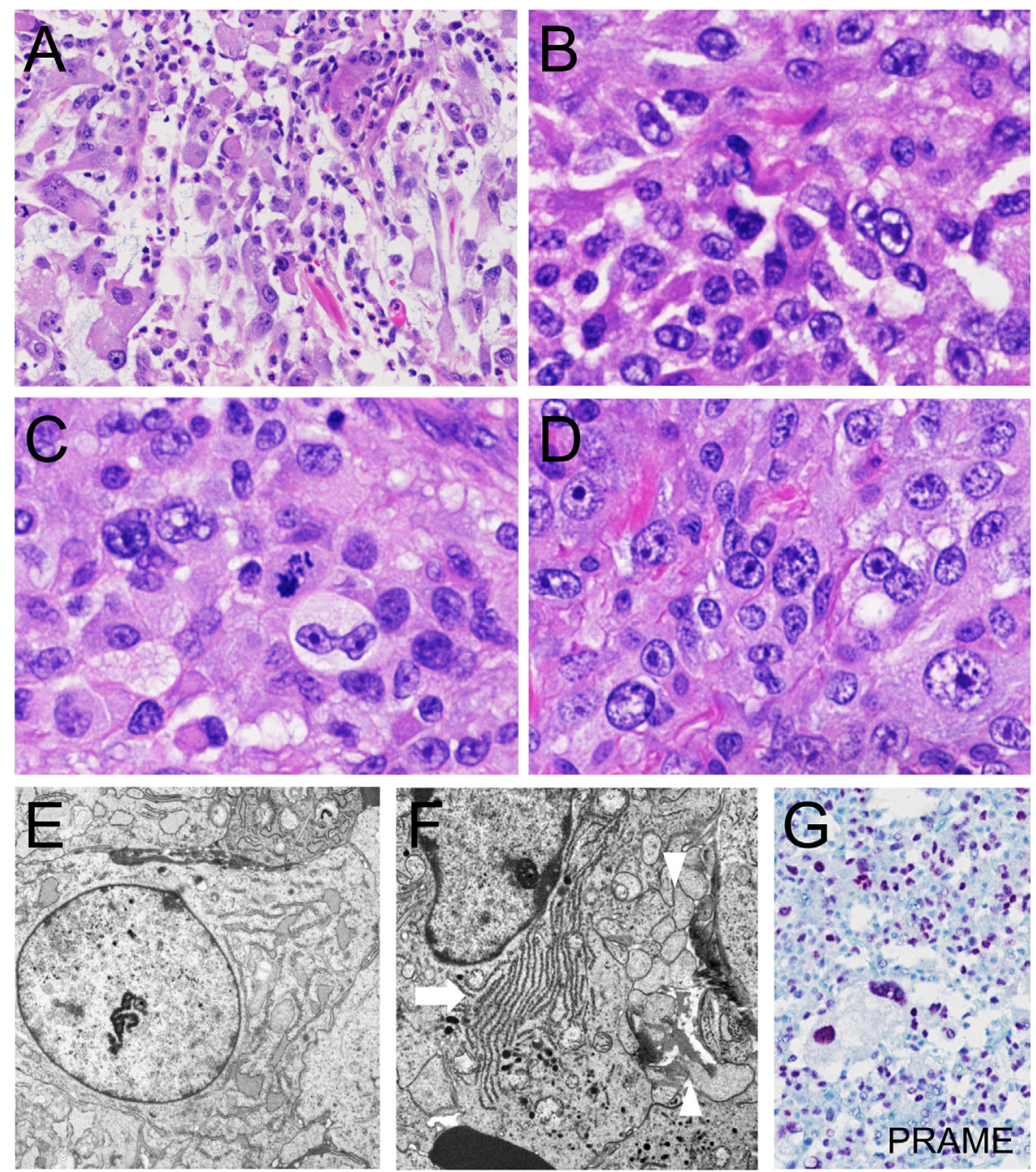

Fig. 2 Myxoinflammatory fibroblastic sarcoma showing (A) ganglion-like cells, and (B-D) virocyte-like cells. (E) Electron microscopy showed tumour cells with abundant dilated rough endoplasmic reticulum, and $(\mathrm{F})$ tumour cells with prominent Golgi apparatus (arrow). Tumour cells showed frequent cell processes intimately associated with collagen fibrils (arrowheads). (G) Tumour cells showed positivity for PRAME immunohistochemistry. 
MyoD1, CD117, PAX8, TTF1, inhibin, HepPar1, arginase, Glypican-3, pan-TRK, NUT, ALK1, ROS1, synaptophysin, chromogranin, and INSM1. Ki-67 labelling index was estimated at up to $30 \%$. Excision of the tumour appeared focally incomplete. Two lymph nodes in the right neck dissection were involved by direct extension.

Electron microscopy on glutaraldehyde-fixed tumour tissue confirmed fibroblastic differentiation. The tumour cells showed abundant cytoplasm which contained prominent, dilated rough endoplasmic reticulum (Fig. 2E). Some cells showed prominent Golgi apparatus (Fig. 2F). The cells had frequent slender cytoplasmic projections intimately associated with collagen fibrils (Fig. 2F). Collections of poorly preserved mitochondria were present in occasional cells. Scattered lysosomes, lipid droplets and glycogen particles were present. Isolated cells contained vague intermediate filaments. No peripheral densities were seen. The nuclei showed prominent nucleoli.

Cytogenetic analysis revealed a complex karyotype including rearrangements of $1 \mathrm{p}$ and $10 \mathrm{q}$ raising the possibility of a $\mathrm{t}(1 ; 10)(\mathrm{p} 22 ; \mathrm{q} 24)$ translocation.

Our case report highlights the diagnostic challenges and potential pitfall for misdiagnosis in a poorly differentiated head and neck high grade epithelioid/sarcomatoid malignancy with PRAME expression. While conventional (low grade) areas were evident on the resection, the initial core biopsies were exclusively composed of the peripheral high grade areas which raised the possibility of malignant melanoma. Interestingly, PRAME expression was observed only in the high grade (poorly differentiated/undifferentiated) areas. This finding, which has not been explicitly noted in previous studies of PRAME-positive high grade MIFS, is in keeping with our current understanding of acral (low grade) MIFS which are negative for PRAME expression. We performed PRAME immunohistochemistry on two historical cases of acral MIFS which were negative (data not shown). PRAME is an immunogenic tumour-associated antigen which is expressed in diverse non-melanocytic epithelial, haematological and soft tissue malignancies. ${ }^{11}$ Its potential as an immunotherapeutic target of prognostic utility awaits further research. ${ }^{12}$

In summary, we present a case of a rare head and neck high grade MIFS in an elderly male patient, which subsequently progressed to multi-site metastatic disease despite adjuvant therapy. High grade MIFS can show PRAME immunoexpression and mimic malignant melanoma. Tissue diagnosis rests on the histological identification of the conventional (low grade) areas which are characteristic, but can be under-sampled in core biopsies.

Acknowledgement: We thank Dr Tristan Rutland for assistance with scanning of digital slides.

Conflicts of interest and sources of funding: The authors state that there are no conflicts of interest to disclose.

Jeremy N. Pulvers ${ }^{1}$, Samuel T. Roberts ${ }^{2,3}$, Andrew Wignall ${ }^{2}$, Renee C. F. Chan ${ }^{4}$, Anita Muljono ${ }^{5}$, Christopher W. Toon ${ }^{1}$

${ }^{1}$ NSW Health Pathology, Department of Anatomical Pathology, Royal North Shore Hospital, Sydney, NSW, Australia; ${ }^{2}$ Department of Otolaryngology, Head and Neck
Surgery, Royal North Shore Hospital, Sydney, NSW, Australia; ${ }^{3}$ University of Newcastle, Callaghan, NSW, Australia; ${ }^{4}$ Department of Anatomical Pathology, Electron Microscopy Unit, Concord Repatriation General Hospital, Sydney, NSW, Australia; ${ }^{5}$ Histopathology Department, Douglass Hanly Moir Pathology, Macquarie Park, NSW, Australia

Contact: Dr Christopher Toon.

E-mail: christopher.toon@health.nsw.gov.au

1. Montgomery EA, Devaney KO, Giordano TJ, et al. Inflammatory myxohyaline tumor of distal extremities with virocyte or ReedSternberg-like cells: a distinctive lesion with features simulating inflammatory conditions, Hodgkin's disease, and various sarcomas. Mod Pathol 1998; 11: 384-91.

2. Meis-Kindblom JM, Kindblom LG. Acral myxoinflammatory fibroblastic sarcoma: a low-grade tumor of the hands and feet. Am J Surg Pathol 1998; 22: 911-24.

3. Michal M. Inflammatory myxoid tumor of the soft parts with bizarre giant cells. Pathol Res Pract 1998; 194: 529-33.

4. Laskin WB, Fetsch JF, Miettinen M. Myxoinflammatory fibroblastic sarcoma: a clinicopathologic analysis of 104 cases, with emphasis on predictors of outcome. Am J Surg Pathol 2014; 38: 1-12.

5. Hallor KH, Sciot R, Staaf J, et al. Two genetic pathways, $\mathrm{t}(1 ; 10)$ and amplification of $3 \mathrm{p} 11-12$, in myxoinflammatory fibroblastic sarcoma, haemosiderotic fibrolipomatous tumour, and morphologically similar lesions. J Pathol 2009; 217: 716-27.

6. Antonescu CR, Zhang L, Nielsen GP, et al. Consistent $\mathrm{t}(1 ; 10)$ with rearrangements of TGFBR3 and MGEA5 in both myxoinflammatory fibroblastic sarcoma and hemosiderotic fibrolipomatous tumor. Genes Chromosomes Cancer 2011; 50: 757-64.

7. Suster D, Michal M, Huang H, et al. Myxoinflammatory fibroblastic sarcoma: an immunohistochemical and molecular genetic study of 73 cases. Mod Pathol 2020; 33: 2520-33.

8. Michal M, Kazakov DV, Hadravsky L, et al. High-grade myxoinflammatory fibroblastic sarcoma: a report of 23 cases. Ann Diagn Pathol 2015; 19: 157-63.

9. Vroobel K, Miah A, Fisher C, et al. Myxoinflammatory fibroblastic sarcoma of the scalp: aggressive behavior at a rare, nonextremity site. Int J Surg Pathol 2015; 23: 292-7.

10. McFarlane R, Meyers AD, Golitz L. Myxoinflammatory fibroblastic sarcoma of the neck. J Cutan Pathol 2005; 32: 375-8.

11. Lezcano C, Jungbluth AA, Nehal KS, et al. PRAME expression in melanocytic tumors. Am J Surg Pathol 2018; 42: 1456.

12. Roszik J, Wang WL, Livingston JA, et al. Overexpressed PRAME is a potential immunotherapy target in sarcoma subtypes. Clin Sarcoma Res 2017; 7: 1-7.

DOI: https://doi.org/10.1016/j.pathol.2021.07.014

\section{Well-differentiated neuroendocrine tumour arising from diffuse ganglioneuromatosis: first reported case}

To the Editor,

This is the first reported case of a well-differentiated neuroendocrine tumour (WDNET) arising from diffuse ganglioneuromatosis in a patient with neurofibromatosis type 1 (NF1 ). The case additionally highlights diffuse neuroendocrine cell micronests, which may represent a potential precursor lesion from which the WDNET arose.

A 51-year-old female with NF-1 underwent a screening colonoscopy, which revealed a $1.8 \mathrm{~cm}$ mass in the upper rectum. Endoscopy revealed a mass on the posterior wall of the upper rectum, suspicious for a neurofibroma. Biopsy revealed a 